\title{
Sistema Classificador de Mapas Conceituais: Uma Arquitetura Computacional Baseada em Processamento de Linguagem Natural
}

\author{
Raimundo N. B. Neto ${ }^{1}$, Rommel W. de Lima ${ }^{2}$ \\ ${ }^{1}$ Universidade do Estado do Rio Grande do Norte - UERN \\ Programa de Pós-Graduação em Ciência da Computação - UERN/UFERSA \\ Caixa Postal 59.610-210 - Mossoró - RN - Brazil \\ \{bezerraneto, rommelwladimir\}@uern.br
}

\begin{abstract}
With the democratization of information, the traditional teaching model gains an even greater challenge, that of keeping students motivated in the classroom and, at the same time, optimizing the teaching-learning process for teachers. The use of Conceptual Maps has been gaining space in the educational environment, allowing the modeling of knowledge through the organization of concepts in a propositional structure. Following these idea, we aim to present a computational architecture focused on the analysis of natural language, which uses the textual relationships produced by Concept Maps and developed in the CmapTools tool to classify the student's cognition level and, additively, expose these results in an analytical way to serve as a subsidy for the teacher.
\end{abstract}

Resumo. Com a democratização da informação, o modelo de ensino tradicional ganha um desafio ainda maior, o de manter os alunos motivados em sala de aula e, no mesmo momento, otimizar o processo de ensino aprendizagem para os professores. A utilização de Mapas Conceituais vem ganhando espaço no ambiente educacional, permitindo a modelagem do conhecimento através da organização de conceitos em uma estrutura proposicional. Seguindo essa ideia, buscamos como objetivo deste trabalho apresentar uma arquitetura computacional focada na análise da linguagem natural, que utiliza os relacionamentos textuais produzidos pelos Mapas Conceituais e desenvolvidos na ferramenta CmapTools para classificar o nível de cognição do aluno e, aditivamente, expor esses resultados de forma analítica para servir de subsídio para o professor.

\section{Introdução}

O ensino superior tradicional vem passando por mudanças estruturais e metodológicas nos últimos tempos. Transformações essas que desafiam a forma de ensinar, de aprender e que atinge diretamente a sua sustentabilidade. Para [Valente 2014] as salas de aula cada vez mais vazias são reflexos da maneira passiva de se lecionar, onde acredita-se que os alunos aprendem no mesmo ritmo e da mesma forma.

Para [Bloom 1944] e [Bloom 1972] a capacidade humana de aprendizagem ocorre de forma diferente entre os indivíduos. Ao longo da história julgou-se que o motivo pelo qual uma porcentagem de discentes atingia uma performance melhor do que outros estava associada às situações e variáveis existentes fora do ambiente educacional o qual, 
IX Congresso Brasileiro de Informática na Educação (CBIE 2020)

Anais do XXXI Simpósio Brasileiro de Informática na Educação (SBIE 2020)

nas mesmas condições de aprendizagem, todos aprenderiam com a mesma capacidade e argúcia o conteúdo.

Além disso, segundo [Valente 2014], o modelo de universidade que faz pesquisa, gera conhecimento e o distribui para poucos, já não se sustenta mais. Nesse sentido, novas metodologias surgem para preencher lacunas deixadas pelos modelos tradicionais, com estratégias que utilizam de tecnologias recentes para proporcionar ambientes de aprendizagem mais flexíveis. Para [Fava 2014], esses novos ambientes geram um ensino mais dinâmico, envolvido em um aprendizado mais ativo.

Seguindo esse propósito, a Organização das Nações Unidas para a Educação, a Ciência e a Cultura (UNESCO) indica a efetiva utilização de ambientes educacionais tecnológicos, trazendo relação direta entre educação, tecnologia e bem-estar social. Continuando nesse contexto [Magrini et al. 2010] afirma que "para viver, aprender e trabalhar bem em uma sociedade cada vez mais complexa, rica em informação e baseada em conhecimento, alunos e professores devem usar a tecnologia de forma efetiva [...]".

Corroborando com o exposto, [Kowata 2010] afirma que o desenvolvimento do conhecimento em um ambiente de ensino e aprendizagem deve ser facilitado, utilizando mecanismos tecnológicos, e sem dissociar o domínio técnico do pedagógico.

Dentro dessa realidade têm surgido diversas propostas de práticas pedagógicas alternativas como por exemplo, as metodologias ativas [Moran 2017]. Os métodos ativos baseia-se em um modelo onde o aluno assume uma postura mais participativa na qual ele resolve problemas, desenvolve projetos e cria oportunidades para a construção do seu conhecimento, possibilitando também uma aprendizagem mais participativa.

Entre as propostas que estão surgindo, alguns propõem o uso dos Mapas Conceituais e da aprendizagem significativa, como descrito por [Barbosa and de Moura 2013], onde afirmam que mapas conceituais caracterizam-se como artifício ativo que faz com que os alunos relacionem o conteúdo e assimilem o conhecimento, conectando novos saberes com os que já possuem, o que possibilita uma aprendizagem mais significante.

Assim sendo, este trabalho descreve uma arquitetura computacional que através do Processamento de Linguagem Natural - PLN e utilizando os conceitos da Aprendizagem Significativa e o uso de Mapas Conceituais, identifica o nível de cognição do aprendiz.

Para isso, além desta introdução, o artigo está estruturado com mais cinco seções. A seção 2 apresenta os conceitos que serviram como base para o desenvolvimento da arquitetura proposta. A seção 3 justifica o objeto da pesquisa. A seção 4 trata da metodologia, fase que define como e onde a pesquisa será realizada, e como será categorizada. A seção 5 aborda a construção da Arquitetura Computacional, evidenciando todos os seus módulos e, por fim, a seção 6 encerra o estudo com os Resultados e Conclusões.

\section{Referencial Teórico}

Esta seção apresenta uma revisão teórica, referenciando e comparando os principais conceitos norteadores da pesquisa. Em síntese abordaremos os princípios da Teoria da Apendizagem Significativa, procurando explorar a relação entre o conhecimento prévio e o novo conhecimento, buscando ainda explicar de que forma esses conceitos se relacionam e se reconectam. Além disso, associaremos a essa ideia os conceitos basilares da utilização de Mapas Conceituais, fazendo a relação dos seus métodos de criação e 
estruturação com a Aprendizagem Significativa. E, por fim, apresentaremos as técnicas de Processamento de Linguagem Natural - PLN voltadas ao reconhecimento de padrões linguisticos que ajudem a identificar as relações gramaticais nas proposições geradas pelos Mapas Conceituais, possibilitando assim classificar esses e auxiliar o professor no processo de ensino aprendizagem.

\subsection{Aprendizagem Significativa}

Contrapondo a Aprendizagem Mecânica [Braathen 2012], que acontece quando o discente introduz um novo conhecimento de forma arbitrária, David Ausubel propôs, à comunidade científica, a Teoria da Aprendizagem Significativa [Ausubel 1963].

A espinha dorsal da Teoria da Aprendizagem Significativa de [Ausubel et al. 1980] refere-se a importância dada aquilo que o indivíduo já conhece, do entendimento já assimilado, ou seja, o conhecimento já existente em sua estrutura cognitiva com compreensão, equilíbrio e diferenciação. Esse conhecimento é chamado pela literatura de subsunçores, sendo necessário, obviamente, investigar previamente tal entedimento para adotar estratégias de ensino adequadas.

Mesmo sendo de meados do século passado, a Teoria da Aprendizagem Significativa continua sendo bastante referenciada em trabalhos recentes, por exemplo: [Jesus and Silva 2004], [Moreira 2006], [Martins et al. 2011], [Moraes et al. 2011], [Braathen 2012] e [dos Santos Lemos 2013].

Com base nesses trabalhos, evidencia-se que a Aprendizagem Significativa continua sendo matéria atual e com espaço para outros trabalhos e novas abordagens. Seguindo essa direção, além desses trabalhos, esta pesquisa utiliza-se de outras referências, como por exemplo: [Lamas et al. 2005], [Kowata et al. 2012], [Lovati et al. 2017] e [Gaspar et al. 2018], para dar um embasamento teórico maior ao estudo, sinalizando a relevância em se utilizar as estratégias participativas em sala de aula, explorando os subsunçores, os conceitos de diferenciação progressiva e reconciliação integrativa [Moreira 2009] e da classificação da taxonomia topológica, associando toda essa sistematização ao nível de aprendizagem adquirido, podendo assim relacionar esses temas com a Aprendizagem Significativa.

\subsection{Mapas Conceituais}

A ideia original dos Mapas Conceituais surge da inquietação do pesquisador norteamericano Joseph Novak em investigar os fatores limitadores do aprendizado em crianças de seis a oito anos. O estudo tinha a pretensão de pesquisar a construção da estrutura cognitiva da criança no processo de aprendizagem [Novak and Cañas 2006].

Um Mapa Conceitual é uma ferramenta utilizada para organizar e representar o conhecimento. A Figura 1 exibe um Mapa Conceitual contendo os seus próprios componentes relacionados [Novak and Cañas 2010]. Observa-se que se trata de um diagrama de conceitos relacionados dentro de um determinado contexto. Os conceitos são representados por caixas e a relação entre os conceitos por frase de ligações. A interligação dos conceitos por frase de ligação recebe o nome de proposição.

Conforme afirma [Kowata 2010], no decorrer da história, o conceito de "Mapas Conceituais" vem sendo empregado sem moderação na literatura, com diversas especificações e formas de uso, confundindo assim sua correta utilização . 


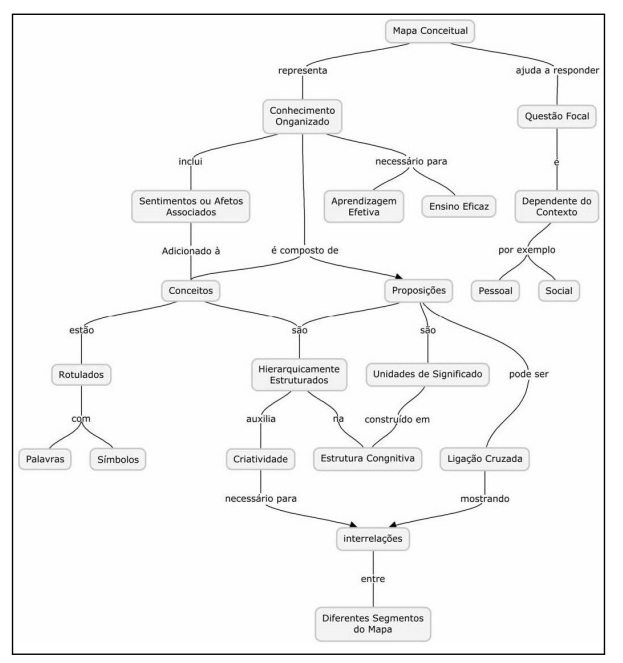

Figura 1. Mapa Conceitual contendo seus conceitos

Este trabalho prioriza o estudo sobre Mapas Conceituais, que por sua vez está diretamente relacionado com a Teoria da Aprendizagem Significativa. Nesse sentido, o Mapa Conceitual de um individuo sobre um determinado contexto, representa a estrutura cognitiva dessa pessoa sobre aquele assunto.

\subsection{Processamento de Linguagem Natural}

O Processamento de Linguagem Natural (PLN) é uma subárea da Inteligência Artificial (IA) responsável por criar modelos computacionais que desempenham tarefas apoiadas em linguagem natural. Segundo [Young et al. 2018], atualmente as pesquisas em PLN então concentradas em abordagens que trabalhem com deep learning [LeCun et al. 2015]. Tratam-se de abordagens que facilitam o processamento ágil de grandes volumes de dados textuais, tendo como propósito minerar informações significativas através da busca de padrões para classificação de textos.

Algoritmos de PLN representam modelos matemáticos voltados ao processamento textual, utilizando-se de bases de dados contextualizada chamada de corpora [Olohan 2004], para aprender computacionalmente. Segundo [Gomes and Evsukoff 2019], essa computação reflete a tentativa de processar e associar características fundamentais de uma linguagem com morfologia, sintaxe e semântica.

A morfologia estuda a arrumação, a composição e a classificação das palavras, reconhecendo-as pela composição das suas unidades primitivas. A Sintaxe considera as regras que constroem as frases nas línguas naturais, ou seja, a parte da gramática. A semântica discute o significado e o atribui as palavras em uma estrutura sintática.

\section{Problemática}

O atual modelo pedagógico utilizado na universidade moderna, onde o professor é mero transmissor de conteúdo está se tornando obsoleto. Sobre isso [Tapscott and Williams 2010] declara que esse padrão baseado na transmissão pode ter sido apropriado para uma economia e uma geração anterior, onde a necessidade e a rapidez da informação seguia outros contornos. Hoje, esse modelo deixa de atender às necessidades de uma nova geração de estudantes que estão imergidos em um cenário global 
do conhecimento, gerando assim um elevado índice de evasão nas instituições de ensino superior no Brasil.

Segundo [Ausubel et al. 1980], o fator isolado mais importante que influencia a aprendizagem é aquilo que o aprendiz já conhece. Compreendendo aquilo que o aluno já conhece sobre o assunto, fica mais simples o planejamento e as ações de ensino do professor.

Em vista disso, faz-se necessário desenvolver um método que consiga reunir a habilidade de compreender o que o discente já conhece sobre o conteúdo e a tradução desse conhecimento impresso nos Mapas Conceituais. Ao mesmo tempo, as Tecnologias Digitais de Informação e Comunicação (TDIC) vem auxiliar essa proposta, possibilitando a criação de novas soluções que unam professores, alunos, pessoas, objetos e informações que estejam envolvidos nesse processo.

Assim sendo, este trabalho descreve o desenvolvimento de uma arquitetura computacional que, utilizando Processamento de Linguagem Natural - PLN, emprega os conceitos da Aprendizagem Significativa e o uso de Mapas Conceituais na visão de [Novak and Cañas 2010] para identificar o nível de cognição do aprendiz através da geração de scores, de acordo com o que teoriza [Cañas et al. 2006] para a estrutura geral dos mapas conceituais.

\section{Metodologia}

Tendo como base o objetivo da pesquisa, que é classificar o nível de cognição do aprendiz a partir de um Mapa Conceitual desenvolvido pelo mesmo sobre um determinado tema, aplica-se a pesquisa bibliográfica para embasar a teoria da aprendizagem significativa auxiliando a desenvolver, explicar e modificar conceitos e ideias.

No que respeita os critérios técnicos, adota-se a pesquisa exploratória visando auxiliar o trabalho com Mapas Conceituais, o Processamento de Linguagem Natural e a validação dos experimentos, utilizando a análise do especialista e a aplicação do teste hipóteses, se propondo a realizar uma análise da literatura existente. Com isso, nosso estudo dispõe-se a buscar metodologias, conceitos e técnicas voltadas a aplicações que estudem, de alguma forma, Mapas Conceituais com eixo em educação utilizando Processamento de Linguagem Natural.

\section{A Proposta}

A arquitetura proposta neste trabalho representa um conjunto de métodos que, fundamentado nas proposições de um Mapa Conceitual criado por um aluno sobre um determinado assunto, classifica esse mapa auxiliando na avaliação da cognição do aluno.

Para realizar essa classificação a arquitetura estrutura uma base de dados específica baseado em um conteúdo previamente proposto. Em seguida as proposições elaboradas na ferramenta CmapTools são exportadas para os módulos da arquitetura, que de forma computacional estrutura, normaliza, valida, compara, nivela e por fim pontua essas proposições. Toda essa sequência de passos retorna uma score que auxiliará o professor a identificar o grau de aprendizado adquirido pelo aluno, através do nível de cognição apresentado pelo mapa. 
A arquitetura proposta é dividia em três módulos e oito passos, conforme ilustrado na Figura 2. O primeiro módulo representa a fase inicial, remete a criação do Mapa Conceitual através do CmapTools e sua posterior exportação do arquivo no formato Outline cmap. O segundo módulo retrata a estruturação da base de dados, resultado da compilação dos textos do material de apoio, é oportuno explicar que a base de dados só iniciará sua interação com o terceiro componente no momento em que o processo do algoritmo de análise contextual for iniciado. O terceiro módulo trata da avaliação das proposições e será detalhado posteriormente.

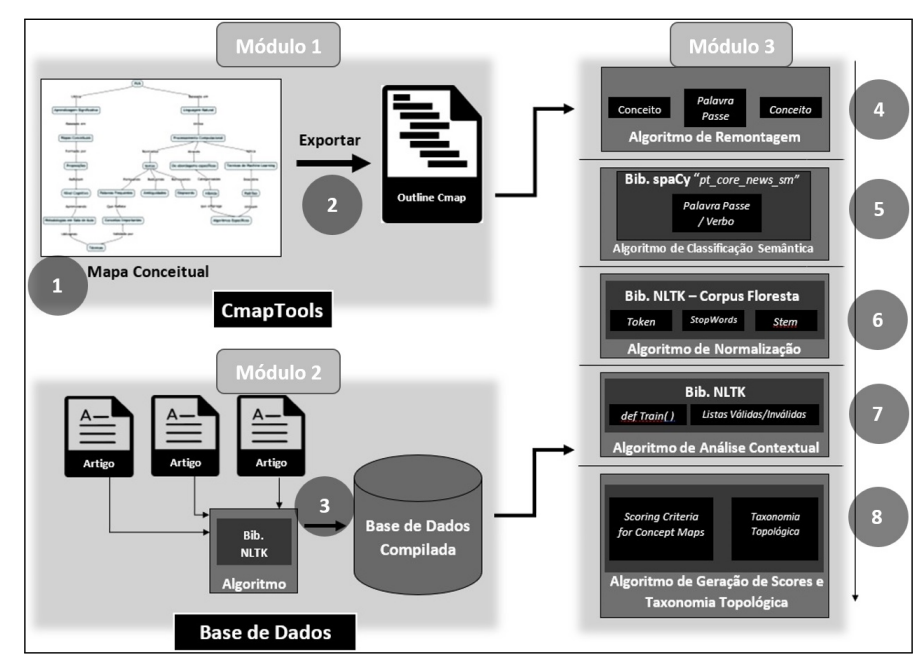

Figura 2. Arquitetura do Sistema

O Módulo 1 é composto pelo "CmapTools" e corresponde aos dois primeiros passos da arquitetura (Figura 2). Trata-se da criação do Mapa Conceitual (passo 1) e a exportação de suas proposições em um arquivo de saída no formato Outline cmap (passo 2).

Para a realização das duas etapas do Módulo 1 é utilizada a ferramenta CmapTools [Cañas et al. 2004], onde o aluno desenvolve o Mapa Conceitual solicitado pelo professor (passo 1) e exporta o mapa no formato Outline cmap (passo 2). É importante explicar que o CmapTools é uma ferramenta de terceiros, e se trata da única parte da arquitetura que não utilizou implementação extra.

O Módulo 2 corresponde ao (passo 3) de geração do corpora. Trata-se de um banco de dados que reúne o material de apoio selecionado pelo professor que é compilado em um único arquivo e ranqueado, gerando assim critérios para a validação das proposições. Após essa etapa, a "Base de Dados" fica em stand by aguardando a interação com os próximos passos.

Por fim, o Módulo 3 é responsável pela validação e classificação das proposições. Esse módulo contém 5 etapas, Figura 2, e tem inicio com o recebimento do arquivo Outline cmap (passo 4). A recepção do arquivo é realizada pelo Algoritmo de Remontagem que irá organizar as informações do arquivo em um modelo específico. Essa "remontagem" planifica o arquivo para a próxima etapa (passo 5) que realizará validação semântica das informações e prepara as proposições para a fase seguinte. A Normalização (passo 6) segmenta as proposições rotulando e adicionando atributos nos tokens, nesse ponto as proposições ganham identidade permitindo assim a sua validação junto a base de dados. 
Após a normalização, há uma interação entre o terceiro e o segundo módulo da arquitetura. Assim no passo 7, o Algoritmo de Análise Contextual realiza inferência no banco de dados e identifica se a proposição está de acordo com as métricas propostas. E, por último, temos a Geração dos Scores e Classificação Topológica do Mapa Conceitual (passo 8), etapa responsável pela pontuação das proposições válidas e inválidas e posterior classificação topológica.

\section{Resultados e Conclusões}

Como mencionado na metodologia, o desenvolvimento da arquitetura proposta partiu de um experimento com uma turma com nove alunos, do [texto oculto para blindagem dos autores] do curso de Graduação em Ciência da Computação para elaboração dos mapas utilizados nesse projeto.

De maneira introdutória, foi ministrada uma aula para apresentar aos alunos os conceitos de MC's, partindo de uma visão holística e indo até as técnicas de escolha e estruturação das proposições. Esse momento serviu para que os aprendizes pudessem tirar suas dúvidas sobre a metodologia e também de como utilizar o CmapTools para construir seus mapas. Ao final da aula, todo o material foi disponibilizado para os alunos, inclusive uma videoaula explicando a instalação e utilização da ferramenta CmapTools em sua versão 6.03 .

Além disso, o tema "Processamento de Linguagem Natural" foi selecionado para servir de base de conhecimento na criação dos mapas, três artigos científicos foram escolhidos para montar o referencial teórico, e com isso auxiliar os alunos no exercício. Ao final da atividade, os alunos foram instruídos a desenvolver o Mapa Conceitual nos moldes da pesquisa, passos 1 e 2 da arquitetura.

Para geração dos resultados, no Módulo 1, foram gerados 9 mapas conceituais produzidos nos passos 1 e 2 da arquitetura. Estes produziram um total de 197 proposições identificadas no passo 4 do Módulo 3 que, após nova validação, passo 5, resultou em 110 proposições preparadas para a análise contextual.

Na sequência, passo 6, as proposições foram subdivididas e classificadas em 1179 tokens e 251 stopwords, tagueamento necessário para a normalização do texto.

No passo 7 as 110 proposições foram processadas para geração de scores conforme estudo de [Gowin and Alvarez 2005]. Por fim no passo 8 usamos a Taxonomia Topológica de [Cañas et al. 2006] para ranquear os Mapas Conceituais.

A Figura 3 reproduz a tabela utilizada para realizar a validação das proposições, onde cada relacionamento foi inserido conforme as ligações impressas no mapa conceitual desenvolvido pelo aluno. Observa-se na figura que a coluna "Análise Ferramenta" é destinada para os resultados obtidos do algoritmos de processamento textual e, a coluna "Análise Especialista" ficou reservada para a validação manual do professor.

A validação ocorreu em três etapas, a primeira foi feita pelo especialista, proposição por proposição, onde o mesmo aprovava ou reprovava os trechos em cada classe, a segunda validação ocorreu com a análise automática da arquitetura proposta, também nas três classes, e por último foi comparado o resultado da etapa 1 com o da etapa 2, gerando uma acurácia de (100\%) na estrutura da proposição, que leva em consideração o modelo CONCEITO 1 - VERBO - CONCEITO 2, de $(78 \%)$ da estrutura gramati- 


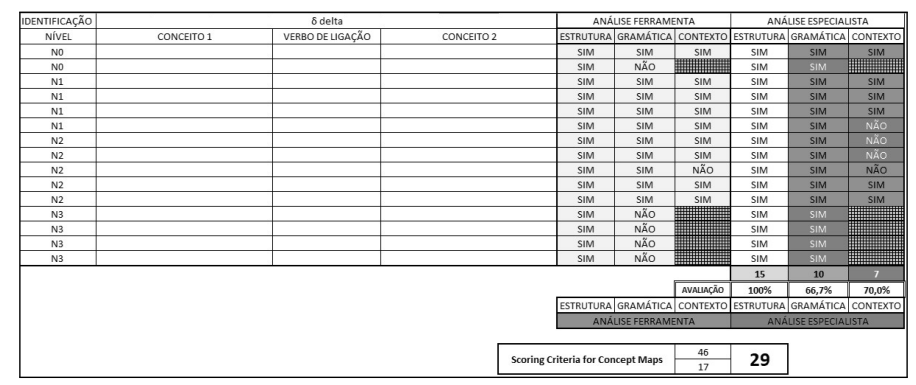

Figura 3. Tabela de Validação das Proposições

cal, que leva em consideração a presença do verbo classificado gramaticalmente e, por fim, $(84 \%)$ de contexto, que avalia se a proposição está de acordo com o conteúdo programático.

As informações sobre a estrutura gramatical e contexto podem ser observados na Figura 4 que mostra a relação das duas referências em todos os 9 mapas conceituais avaliados nesse trabalho. Essa análise é provada seguindo a métrica do teste de hipótese ${ }^{1}$, que coleta evidências nos dados amostrais para fundamentar a refutação ou não de uma certa alegação, conforme afirma [Naghettini and Pinto 2007]. Com o experimento validado e os resultados obtidos, o trabalho se revela condizente com o que se propõe.

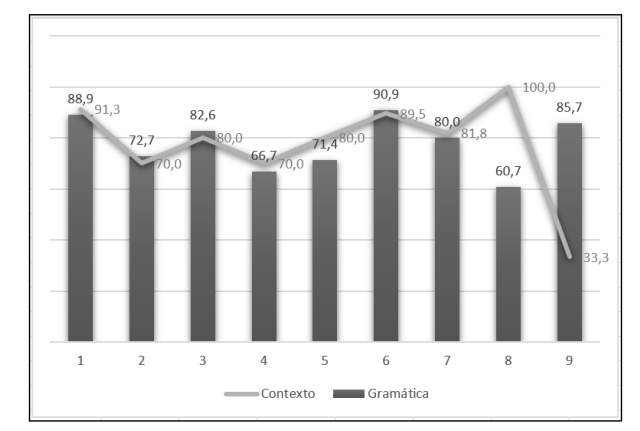

Figura 4. Estrutura Gramatical e Contexto

Esse trabalho permitiu compreender a relação entre a Classificação de Mapas Conceituais e a influência desses na Aprendizagem Significativa, dessa forma percebemos a real necessidade de utilizar uma arquitetura que pudesse mensurar esses mapas e categorizar essa avaliação para retrata a compreensão do aluno sobre um determinado tema.

Em síntese, foi desenvolvida uma arquitetura computacional que analisa as proposições com o objetivo de classificar Mapas Conceituais, relacionado essa classificação aos conceitos de Aprendizagem Significativa. Sob uma ótica computacional, consideramos que o estudo foi bem-sucedido, na medida em que especificou e validou cada processo da arquitetura. Sob um contexto mais amplo e em consonância com os exemplos elencados em nosso referencial, há de se considerar que as respostas aos assuntos principais da pesquisa foram atendidas, comprovando assim a sua viabilidade.

\footnotetext{
${ }^{1}$ Teste de Hipóteses Estatística é uma metodologia que assessora a tomada de decisões e assegura escolher entre a hipótese nula $\mathrm{HO}$ ou hipótese alternativa Ha, baseando-se na amostra.
} 
IX Congresso Brasileiro de Informática na Educação (CBIE 2020)

Anais do XXXI Simpósio Brasileiro de Informática na Educação (SBIE 2020)

\section{Referências}

Ausubel, D. P. (1963). The psychology of meaningful verbal learning.

Ausubel, D. P., Novak, J. D., and Hanesian, H. (1980). Psicologia educacional. Interamericana.

Barbosa, E. F. and de Moura, D. G. (2013). Metodologias ativas de aprendizagem na educação profissional e tecnológica. Boletim Técnico do Senac, 39(2):48-67.

Bloom, B. S. (1944). Some major problems in educational measurement. Journal or Educational Research, 38(1):139-142.

Bloom, B. S. (1972). Innocence in education. The School Review, 80(3):333-352.

Braathen, P. C. (2012). Aprendizagem mecânica e aprendizagem significativa no processo de ensino-aprendizagem de química. Revista eixo, 1(1):63-69.

Cañas, A. J., Hill, G., Carff, R., Suri, N., Lott, J., Gómez, G., Eskridge, T. C., Arroyo, M., and Carvajal, R. (2004). Cmaptools: A knowledge modeling and sharing environment.

Cañas, A. J., Novak, J. D., Miller, N. L., Collado, C., Rodríguez, M., Concepción, M., Santana, C., and Peña, L. (2006). Confiabilidad de una taxonomía topológica para mapas conceptuales.

dos Santos Lemos, E. (2013). A aprendizagem significativa: estratégias facilitadoras e avaliação. Série-Estudos-Periódico do Programa de Pós-Graduação em Educação da $U C D B,(21)$.

Fava, R. (2014). Educação 3.0: aplicando o pdca nas instituições de ensino. São Paulo: Saraiva, page 256.

Gaspar, W., Aguiar, C., Gava, T., and Cury, D. (2018). Uma arquitetura tecnológica para apoiar o professor na utilização de mapas conceituais em sala de aula. In Brazilian Symposium on Computers in Education (Simpósio Brasileiro de Informática na Educação-SBIE), volume 29, page 158.

Gomes, D. d. S. M. and Evsukoff, A. G. (2019). Processamento de linguagem natural em português e aprendizagem profunda para o domínio de óleo e gás. arXiv, pages arXiv-1908.

Gowin, D. B. and Alvarez, M. C. (2005). The art of educating with V diagrams. Cambridge University Press.

Jesus, M. d. and Silva, R. C. O. (2004). A teoria de david ausubel-o uso dos organizadores prévios no ensino contextualizado de funções. VII Encontro Nacional de Educação Matemática-2004.

Kowata, J. H. (2010). Uma abordagem para construção automática de mapas conceituais a partir de textos em língua portuguesa (brasil). Master's thesis, Universidade Federal do Espírito Santo.

Kowata, J. H., Cury, D., and Boeres, M. C. S. (2012). Em direção à construção automática de mapas conceituais a partir de textos. Revista Brasileira de Informática na Educação, 20(1):33. 
IX Congresso Brasileiro de Informática na Educação (CBIE 2020)

Anais do XXXI Simpósio Brasileiro de Informática na Educação (SBIE 2020)

Lamas, F., Boeres, C., Cury, D., and de Menezes, C. S. (2005). Comparando mapas conceituais utilizando correspondência de grafos. In Brazilian Symposium on Computers in Education (Simpósio Brasileiro de Informática na Educação-SBIE), volume 1, pages 24-27.

LeCun, Y., Bengio, Y., and Hinton, G. (2015). Deep learning. nature, 521(7553):436444.

Lovati, M., Cury, D., Aguiar, C., and Azeredo, R. (2017). Clusterizando mapas conceituais para identificar desempenho cognitivo em grupos. In Brazilian Symposium on Computers in Education (Simpósio Brasileiro de Informática na Educação-SBIE), volume 28, page 1397.

Magrini, D. F., dos Anjos Pereira, D., de Souza Zacarias, P. H., and Young, C. R. (2010). Organização das nações unidas para educação, ciência e cultura (unesco).

Martins, R. L. C., Linhares, M. P., and Reis, E. M. (2011). Mapas conceituais como instrumento de avaliação e aprendizagem de conceitos físicos sobre mecânica do vôo. Revista Brasileira de pesquisa em Educação em Ciências, 9(1).

Moraes, J. U. P., Santana, R., and Viana-Barbosa, C. J. (2011). Avaliação baseada na aprendizagem significativa por meio de mapas conceituais. Atas do VIII ENPEC, Campinas.

Moran, J. (2017). Metodologias ativas e modelos híbridos na educação. S. YAEGASHI e outros (Orgs). Novas Tecnologias Digitais: Reflexões sobre mediação, aprendizagem e desenvolvimento. Curitiba: $C R V$, pages 23-35.

Moreira, M. A. (2006). Aprendizagem significativa: da visão clássica à visão crítica (meaningful learning: from the classical to the critical view). In Conferência de encerramento do V Encontro Internacional sobre Aprendizagem Significativa, Madrid, Espanha, setembro de.

Moreira, M. A. (2009). Subsídios teóricos para o professor pesquisador em ensino de ciências: A teoria da aprendizagem significativa. Porto Alegre-RS.

Naghettini, M. and Pinto, É. J. d. A. (2007). Hidrologia estatística. CPRM.

Novak, J. D. and Cañas, A. J. (2006). The origins of the concept mapping tool and the continuing evolution of the tool. Information visualization, 5(3):175-184.

Novak, J. D. and Cañas, A. J. (2010). A teoria subjacente aos mapas conceituais e como elaborá-los e usá-los. Práxis Educativa, 5(1):9-29.

Olohan, M. (2004). Introducing corpora in translation studies. Routledge.

Tapscott, D. and Williams, A. D. (2010). Innovating the 21 st-century university: It's time. Educause review, 45(1):16-29.

Valente, J. A. (2014). Blended learning e as mudanças no ensino superior: a proposta da sala de aula invertida. Educar em Revista, (SPE4):79-97.

Young, T., Hazarika, D., Poria, S., and Cambria, E. (2018). Recent trends in deep learning based natural language processing. ieee Computational intelligenCe magazine, 13(3):55-75. 